\title{
Pretreatment Pokemon Level as a Predictor of Response to Cisplatin and Paclitaxel in Patients with Unresectable Non-Small Cell Lung Cancer
}

\author{
Quan-Le Zhang ${ }^{a} \quad$ Xi-Zhi Xing ${ }^{a} \quad$ Feng-Yan Li ${ }^{b} \quad$ Ya-Juan Xing ${ }^{a} \quad$ Jing Li $^{\mathrm{a}}$ \\ a Division of Respiratory Medicine, Cangzhou Hospital of Integrated Traditional Chinese and Western Medicine of Hebei Province, \\ Cangzhou, China; \\ ${ }^{\mathrm{b}}$ Radiology Department, Cangzhou Hospital of Integrated Traditional Chinese and Western Medicine of Hebei Province, Cangzhou, China
}

Keywords

Pokemon · NSCLC $\cdot$ Chemotherapy

\section{Summary}

Background: We firstly investigated the expression of Pokemon in patients with non-small cell lung cancer (NSCLC), then characterized the role of Pokemon in evaluating the response to combined cisplatin and paclitaxel chemotherapy and prognosis. Patients and Methods: In this study, 61 patients with previously untreated locally advanced or metastatic NSCLC were treated with a combination chemotherapy comprising cisplatin and paclitaxel. The correlation between serum expression of Pokemon and effectiveness of chemotherapy was assessed. Results: The expression level of Pokemon in NSCLC patients was higher than that in healthy controls $(p=0.000)$, and was correlated with tumor size and TNM stage $(p<0.05)$. Kaplan-Meier analysis and Cox proportional hazard model demonstrated a poor response and shorter survival time in patients with pretreatment Pokemon levels in excess of $135.09 \mathrm{ng} / \mathrm{ml}$ compared to those with Pokemon levels below $135.09 \mathrm{ng} / \mathrm{ml}$ ( $p=0.013$ ). Pokemon $\geq 135.09 \mathrm{ng} / \mathrm{ml}$ was an independent risk factor for survival time in NSCLC patients undergoing combination chemotherapy $(p=0.018)$. Conclusion: The serum level of Pokemon correlated with efficacy of cisplatin and paclitaxel combination chemotherapy and survival time, which indicated that Pokemon may be a potentially useful biomarker for predicting treatment effectiveness of first-line chemotherapy and prognosis in NSCLC.

(c) 2015 The Author(s). Published by S. Karger GmbH, Freiburg

\section{KARGER

Introduction

Pokemon, also known as LRF, OCZF, or FBI-1, is a member of the POK family of transcriptional repressors, which consists of an $\mathrm{NH}_{2}$-terminal POZ/BTB domain and $4 \mathrm{COOH}$-terminal krüppeltype zinc fingers. The POZ/BTB domain is involved in homodimerization or heterdimerization, and recruits some corepressors such as BcoR, NcoR, or SMRT, while the krüppel-type zinc finger domain mediates specific DNA recognition and binding [1-4].

Maeda et al. $[1,2]$ demonstrated in vitro that the deletion of Pokemon in mouse embryofibroblasts can render them almost completely resistant to proliferation and transformation by oncogenic combinations such as adenovirus $\mathrm{E} 1 \mathrm{~A}+\mathrm{H}$-ras, $\mathrm{Myc}+\mathrm{H}$-ras, $\mathrm{T}$ antigen $+\mathrm{H}$-ras, or BCL6+H-ras; however, this resistance can be reversed by coinfection with the Pokemon gene. The transforming potential of Pokemon was further confirmed in vivo in transgenic mice overexpressing Pokemon in immature $\mathrm{T}$ and $\mathrm{B}$ lymphoid cells which formed fatal thymic lymphomas. Therefore, Pokemon, as a vital oncogene, may play important roles in tumorigenesis.

Recent studies have indicated that Pokemon was overexpressed in some human tumors such as breast cancer, colorectal cancer, prostate cancer, bladder cancer, and liver cancer [5-9]. Previous studies also revealed that Pokemon was highly expressed in nonsmall cell lung cancer (NSCLC) tissues $[10,11]$. However, Pokemon serum levels in NSCLC and their clinical importance remain unclear. Since the prognosis for patients with unresectable advanced or metastatic NSCLC undergoing chemotherapy is generally poor, it would be beneficial to identify biomarkers that could predict objective responses to chemotherapy and prognosis in NSCLC patients. Hence, we carried out the following study in order to further explore the role of Pokemon in NSCLC and identify whether it may be a suitable biomarker for NSCLC patients in evaluating chemotherapy response and prognosis.

Quan-le Zhang, MD

Division of Respiratory Medicine, Cangzhou Hospital

of Integrated Traditional Chinese and Western Medicine of Hebei Province

Qiantong Road 17, Cangzhou 061000, China

zhangquanle2006@126.com 
In this study, we examined the serum expression of Pokemon in patients with NSCLC by enzyme-linked immunosorbent assay, and explored its clinical significance in correlation with clinical characteristics of the patients. In a second step, we investigated the association between pretreatment Pokemon levels and response to cisplatin and paclitaxel chemotherapy as well as prognosis in NSCLC patients.

\section{Patients and Methods}

\section{Patients and Treatment Schedule}

Between July 2009 and January 2013, 61 patients with chemonaive unresectable primary NSCLC were enrolled in this study. Inclusion criteria were: i) age $\geq 55$ years and an Eastern Cooperative Oncology Group performance status(ECOG PS) of 0-2; ii) histologically confirmed NSCLC (specimens were derived from sputum, bronchial biopsy, or pleural effusion); iii) stage IIIb-IV according to the TNM staging system of the Union for International Cancer Control (UICC, 7th edition); iv) relatively favorable hematological parameters (hemoglobin concentration $\geq 9.0 \mathrm{~g} / \mathrm{dl}$, absolute neutrophil count $\geq 1,500 / \mathrm{ml}$, and platelet count $\geq 100,000 / \mathrm{ml}$ ), renal function (creatinine clearance $\geq 45$ $\mathrm{ml} / \mathrm{min}$ ), and liver function (total bilirubin $\leq 1.7 \mathrm{mg} / \mathrm{dl}$, transaminase level less than twice the upper limit of normal; and v) $\geq 1$ bidimensionally measurable lesion conforming to the Response Evaluation Criteria in Solid Tumors (RECIST, version 1.1).

Exclusion criteria included any of the following: i) sepsis; ii) coma; iii) uncontrolled congestive heart failure, respiratory failure, hypertension; iv) pregnancy; v) previous chemotherapy or radiotherapy within the last 3 months; vi) uncontrolled diabetes mellitus and/or relatively serious complication(s); and vii) reluctance to cooperate.

All patients received cisplatin and paclitaxel combination chemotherapy in 1-4 cycles (cisplatin, $40 \mathrm{mg} / \mathrm{m}^{2}$, day 1; paclitaxel, $140 \mathrm{mg} / \mathrm{m}^{2}$, days $1-3$; every 4 weeks). Treatment was discontinued at any time in the case of disease progression or unacceptable toxicity or at the patient's request. None of patients received additional treatment such as radiotherapy during or after chemotherapy.

During treatment, a chest computed tomography scan was performed every 4 weeks before each cycle to document disease progression. Objective tumor responses to treatment were evaluated based on RECIST criteria (complete remission (CR), partial response (PR), stable disease (SD), progressive disease $(\mathrm{PD})$ ). The response rate (RR) was defined as the ratio of the number of patients who achieved CR or PR to the number of enrolled patients. Overall survival (OS) was calculated from the start of therapy to disease progression or death, or to the last follow-up. As a control group, 60 healthy subjects were also enrolled in this study. The study protocol was approved by the ethics committee of Cangzhou Hospital of Integrated Traditional Chinese and Western Medicine. All participants gave written informed consent before enrolment in the study.

\section{Measurement of Pokemon}

The serum levels of Pokemon 1 day before treatment or 1 month after chemotherapy were examined by enzyme-linked immunosorbent assay. The main procedure was as follows: approximately $5 \mathrm{ml}$ of venous blood were drawn from the study participants. Blood samples were left overnight at room temperature to allow clotting, and centrifuged at 2,000 rpm for $10 \mathrm{~min}$. The serum was collected in Eppendorf tubes, and stored at $4{ }^{\circ} \mathrm{C}$. Serum samples were tested for anti-Pokemon IgG antibodies using a commercially available enzyme immunoassay kit (Yanjing Biotech, Inc., Shanghai, China). Absorbance of each sample was measured at $490 \mathrm{~nm}$ with a microplate reader (Bio-Rad Laboratories, Inc., CA, USA). The assay was done in triplicate wells, and each experiment was repeated 3 times. All tests were performed following the instructions of the manufacturer.

\section{Statistical Analysis}

Data are shown as mean \pm standard error (SE). The significance of the difference between patients and controls was determined by unpaired t-test, whereas the significance of the difference in patients pre- and post-treatment was judged by paired t-test. OS was assessed using the Kaplan-Meier method, and the 95\% confidence interval (CI) for the median time to events was computed. Univariate analyses were performed by means of the log-rank test. Multivariate analyses were performed with the Cox proportional hazards model. All statistical analyses were performed with SPSS 19.0 for windows (IBM Inc., Armonk, NY, USA). A p value of less than 0.05 was considered to be significant.

\section{Results}

\section{Pokemon Level in Patients and Controls}

In the 61 NSCLC patients analyzed, the serum expression level of Pokemon was $189.66 \pm 91.14 \mathrm{ng} / \mathrm{ml}$ which was higher than that in healthy subjects $(21.50 \pm 14.88 \mathrm{ng} / \mathrm{ml})(\mathrm{t}=10.791, \mathrm{p}=0.000) .52$ (85.2\%) patients showed positive serum expression of Pokemon compared to only $17(28.3 \%)$ healthy subjects. There was a significant difference between the 2 groups $\left(\chi^{2}=3.986 ; p=0.017\right)$.

\section{Pokemon Level and Patients' Baseline Clinical Characteristics}

We then analyzed the associations between Pokemon level and baseline clinicopathological features of the patients. Among various factors, Pokemon was significantly associated with tumor size and TNM stage. No associations were observed between Pokemon and other clinical characteristics (table 1).

\section{Treatment Response}

All patients were evaluable for survival on an intent-to-treat basis, and 55 patients were evaluable for response to treatment. The number of overall response was 39, whereas 9 patients showed SD and 7 patients had PD. The RR was 63.9\% (95\% CI 54.4-59.3\%) (table 2).

\section{Pokemon Level and Response to Chemotherapy}

A receiver operator characteristic (ROC) curve was generated according to patients' treatment responses to chemotherapy (fig. 1, online supplemental www.karger.com/?DOI=440790). As shown in figure 1, the area under the ROC curve was 0.831 (95\% CI $0.675-$ 0.987 ), and the cut-off value was set at $135.09 \mathrm{ng} / \mathrm{ml}$ when the sensitivity was $86.7 \%$ and the specificity was $60.0 \%$. Patients were then classified into 2 groups according to the Pokemon cut-off value: 34 patients had Pokemon levels below $135.09 \mathrm{ng} / \mathrm{ml}$, and 21 patients had Pokemon levels greater than $135.09 \mathrm{ng} / \mathrm{ml}$. In the 34 patients with Pokemon levels $<135.09 \mathrm{ng} / \mathrm{ml}$, the average difference in the level pre- and post-treatment was $35.17 \pm 20.52 \mathrm{ng} / \mathrm{ml}$ which was higher than that in the 21 patients with Pokemon levels $\geq 135.09 \mathrm{ng} / \mathrm{ml}(18.04 \pm 13.89 \mathrm{ng} / \mathrm{ml})(\mathrm{t}=2.192 ; \mathrm{p}=0.023)$. The $\mathrm{RR}$ in patients with Pokemon levels $<135.09 \mathrm{ng} / \mathrm{ml}$ was $69.2 \%$ while it was $30.8 \%$ in patients with Pokemon levels $\geq 135.09 \mathrm{ng} / \mathrm{ml}$. There was a significant difference between the 2 groups $\left(\chi^{2}=6.467\right.$; $\mathrm{p}=0.011)$ (table 3$)$. 
Table 1. Baseline clinicopathological features in 61 patients with non-small cell lung cancer (NSCLC)

\begin{tabular}{|c|c|c|c|c|c|}
\hline Classification & & Patients, $\mathrm{n}$ & $\begin{array}{l}\text { Pokemon expression } \\
\text { level, } \mathrm{ng} / \mathrm{ml}\end{array}$ & $\mathrm{t}$ value & $\mathrm{p}$ value \\
\hline \multirow[t]{2}{*}{ Sex } & male & 48 & $222.04 \pm 114.24$ & 1.514 & 0.141 \\
\hline & female & 13 & $170.91 \pm 71.56$ & & \\
\hline \multirow[t]{2}{*}{ Age, years } & $\geq 60$ & 47 & $195.23 \pm 92.60$ & 0.434 & 0.668 \\
\hline & $<60$ & 14 & $180.04 \pm 92.16$ & & \\
\hline \multirow[t]{2}{*}{ Smoking history } & yes & 53 & $175.39 \pm 75.68$ & -0.854 & 0.401 \\
\hline & no & 8 & $203.93 \pm 105.08$ & & \\
\hline \multirow[t]{2}{*}{ Tumor size, $\mathrm{cm}$} & $\geq 5.0$ & 50 & $213.67 \pm 92.07$ & 2.624 & 0.014 \\
\hline & $<5.0$ & 11 & $123.64 \pm 46.76$ & & \\
\hline \multirow[t]{2}{*}{ Histological type } & $\begin{array}{l}\text { squamous cell } \\
\text { carcinoma }\end{array}$ & 43 & $170.91 \pm 71.55$ & -1.514 & 0.141 \\
\hline & $\begin{array}{l}\text { non-squamous } \\
\text { cell carcinoma }\end{array}$ & 18 & $222.04 \pm 114.24$ & & \\
\hline \multirow[t]{2}{*}{ TNM stage } & IIIB & 38 & $122.51 \pm 42.02$ & -3.673 & 0.001 \\
\hline & IV & 23 & $228.54 \pm 89.72$ & & \\
\hline \multirow[t]{3}{*}{ Differentiation degree } & high & 18 & $216.61 \pm 104.44$ & 0.975 & 0.342 \\
\hline & moderate & 12 & $178.95 \pm 98.39$ & & \\
\hline & low & 31 & $177.35 \pm 75.56$ & & \\
\hline
\end{tabular}

\section{Pokemon Level and Overall Survival}

The mean OS of the 34 patients with Pokemon $<135.09 \mathrm{ng} / \mathrm{ml}$ was 12.7 months (95\% CI 10.3-15.0), while the OS of patients with Pokemon $\geq 135.09 \mathrm{ng} / \mathrm{ml}$ was 9.2 month (95\% CI 7.2-11.2) (table 4). Log-rank test analysis demonstrated that the OS of patients with Pokemon $<135.09 \mathrm{ng} / \mathrm{ml}$ was significantly longer than that of patients with Pokemon $\geq 135.09 \mathrm{ng} / \mathrm{ml}$ ( $\mathrm{p}=0.013$ ) (fig. 2, online supplemental www.karger.com/?DOI=440790). To determine independent prognostic factors, we used the Cox proportional hazards model for multivariate analysis. It demonstrated that the pretreatment Pokemon level was an independent prognostic factor for NSCLC patients (table 5).

\section{Discussion}

The most important findings of our study are i) that NSCLC patients had higher Pokemon serum levels than healthy controls; ii) that patients with pretreatment Pokemon levels $\geq 135.09 \mathrm{ng} / \mathrm{ml}$ had a poor response to cisplatin and paclitaxel combination chemotherapy and a worse prognosis; and iii) that Pokemon is as an independent prognostic factor according to multivariate analysis (Cox proportional hazards model). We also demonstrated that Pokemon expression levels were significantly associated with tumor size and TNM stage, but not with any other clinical characteristics such as smoking history, histological type, or degree of differentiation.

Pokemon, also called FBI-1, LRF, OCZF, was found to be highly expressed in hepatocellular carcinoma, prostate cancer, bladder cancer, and NSCLC, among others. Apostolopoulou et al. [10] observed, in the majority of examined tumor tissue samples, prominent expression of Pokemon in the cancerous areas compared to negligible expression in the adjacent normal tissue sections; also, expression levels were associated with proliferation and tumor size. Zhao et al. [11] demonstrated that the survival rate of NSCLC pa-
Table 2. Treatment response

\begin{tabular}{lc}
\hline Response & Patients, $\mathrm{n}(\%)$ \\
\hline Complete response & $2(3.3)$ \\
Partial response & $37(60.7)$ \\
Stable disease & $9(14.8)$ \\
Progressive disease & $7(11.5)$ \\
Not evaluable & $6(9.8)$ \\
\hline
\end{tabular}

Table 3. Differences in Pokemon levels pre- and post-treatment and response rate

\begin{tabular}{llllll}
\hline Index & Group & & $\mathrm{t}$ & $\chi^{2}$ & $\mathrm{p}$ \\
\cline { 2 - 4 } & $\begin{array}{l}\text { Pokemon } \\
<135.09\end{array}$ & $\begin{array}{l}\text { Pokemon } \\
\geq 135.09\end{array}$ & & & \\
\hline $\begin{array}{r}\text { Difference in } \\
\begin{array}{l}\text { Pokemon, } \\
\text { ng/ml }\end{array}\end{array}$ & $35.17 \pm 20.52$ & $18.04 \pm 13.89$ & 2.192 & - & 0.023 \\
$\mathrm{RR}, \%$ & 69.2 & 30.8 & - & & \\
\hline
\end{tabular}

Table 4. Kaplan-Meier survival analysis for overall survival

\begin{tabular}{llll}
\hline Groups & $\begin{array}{l}\text { Mean overall survival, } \\
\text { months }\end{array}$ & $95 \%$ CI & $\begin{array}{l}\text { Effectiveness } \\
\text { rate, \% }\end{array}$ \\
\hline Pokemon $<135.09^{\mathrm{a}}$ & 12.7 & $10.3-15.0$ & 69.2 \\
& 9.2 & $7.2-11.2$ & 30.8 \\
Pokemon $\geq 135.09$ & 11.5 & $9.7-13.2$ & - \\
All patients & & & \\
\hline
\end{tabular}

${ }^{\mathrm{a}} \mathrm{p}<0.05$ (Pokemon $<135.09$ vs. $\geq 135.09$ ).

$\mathrm{CI}=$ Confidence interval.

tients with negative tissue expression of Pokemon was higher than that of patients with positive Pokemon expression, and it was identified as an independent prognostic factor. 
Table 5. Multivariate analysis using the Cox proportional hazards model

\begin{tabular}{|c|c|c|c|}
\hline Factors & Relative risk & $\begin{array}{l}95 \% \\
\text { confidence } \\
\text { value }\end{array}$ & $\mathrm{p}$ value \\
\hline Sex (male vs. female) & 1.675 & $0.437-5.930$ & 0.424 \\
\hline Age (<60 vs. $\geq 60$ years $)$ & 1.253 & $0.418-3.756$ & 0.687 \\
\hline Smoking history (yes vs. no) & 3.298 & $0.956-11.380$ & 0.059 \\
\hline $\begin{array}{l}\text { Histological type (squamous } \\
\text { cell carcinoma vs. non- } \\
\text { squamous cell carcinoma) }\end{array}$ & 0.726 & $0.104-5.082$ & 0.747 \\
\hline TNM stage (IIIB vs. IV) & 0.479 & $0.041-5.627$ & 0.158 \\
\hline $\begin{array}{l}\text { Differentiation degree } \\
\text { (low vs. high and moderate) }\end{array}$ & 2.367 & $0.526-10.658$ & 0.262 \\
\hline $\begin{array}{l}\text { Lymph node metastasis } \\
\text { (no vs. yes) }\end{array}$ & 0.485 & $0.150-1.565$ & 0.026 \\
\hline $\begin{array}{l}\text { Pokemon } \\
\qquad(<135.09 \text { vs. } \geq 135.09)\end{array}$ & 0.621 & $0.204-1.895$ & 0.018 \\
\hline
\end{tabular}

Although some studies have explored the role of Pokemon in NSCLC, the serum expression of Pokemon has remained unclear. In this study, we firstly explored the Pokemon serum levels in patients with NSCLC, and observed that the total expression levels of patients were higher than those of healthy subjects. However, because of its non-selective expression in patients and controls, it is essential to determine a cut-off level conducive to diagnosing NSCLC. Although our study was limited due to the small sample size, to our knowledge, this was the first analysis of Pokemon levels in patients with NSCLC. Furthermore, through analyzing the relationship between Pokemon expression and patients' baseline clinicopathological features, we showed correlations with tumor size and TNM stage while no associations were observed between Pokemon and other clinical characteristics, which is partly in concert with the result of Apostolopoulou et al. [10].

It is well established that combination chemotherapy can prolong the survival time of patients with NSCLC. However, only few biomarkers are being used for monitoring the efficacy of chemotherapy and evaluating prognosis. In this study, to explore the response to chemotherapy and the survival time of patients with NSCLC, we first determined a cut-off value for the Pokemon serum level, which was calculated based on a ROC curve according to prechemotherapy Pokemon levels. The cut-off value was defined as $135.09 \mathrm{ng} / \mathrm{ml}$ associated with higher specificity and sensitivity. Patients were divided into 2 groups according to the cut-off value: Pokemon $<135.09 \mathrm{ng} / \mathrm{ml}$ and Pokemon $\geq 135.09 \mathrm{ng} / \mathrm{ml}$. Log-rank test analysis demonstrated that the OS of patients with Pokemon $<135.09 \mathrm{ng} / \mathrm{ml}$ was significantly longer than that of those with Pokemon $\geq 135.09 \mathrm{ng} / \mathrm{ml}$. Chi-squared test showed that the effectiveness of chemotherapy in patients with Pokemon $<135.09 \mathrm{ng} / \mathrm{ml}$ was higher than that in patients with Pokemon $\geq 135.09 \mathrm{ng} / \mathrm{ml}$. Finally, to determined independent prognostic factors, multivariate analysis was performed with the Cox proportional hazards model. It was demonstrated that the pretreatment Pokemon level was an independent prognostic factor for NSCLC patients. Hence, Pokemon may serve as a useful serum marker to monitor or assess NSCLC patients' responses to combined chemotherapy.
The present study had some limitations. Firstly, combination chemotherapy consisting of cisplatin and paclitaxel was chosen as the treatment regimen for the 2 following reasons: i) Cisplatin and paclitaxel combination chemotherapy can prolong survival time; a study carried out by a multi-institutional cooperative group in chemonaive stage IIIB-IV NSCLC patients showed superior survival with combined cisplatin and paclitaxel (median survival time, 9.9 months; 1-year survival rate, 38.9\%) compared with cisplatin plus etoposide (median survival time, 7.6 months; 1-year survival rate, $31.8 \%)(\mathrm{p}=0.048)$ while toxicity was similar in the 2 groups [12]; ii) Both drugs are relatively inexpensive which ensures continuation of chemotherapy for less wealthy patients. However, combination chemotherapy consisting of cisplatin and paclitaxel is not a standard treatment in non-squamous NSCLC. For epidermal growth factor receptor (EGFR) mutation(+) NSCLC adenocarcinoma, EGFR inhibitor therapy is the first-line treatment with a significant survival advantage over cisplatin-based chemotherapy. Thus, patients with non-squamous EGFR mutation(+) adenocarcinomas were excluded from the present study.

Secondly, the study demonstrated Pokemon to be a potentially useful biomarker for predicting treatment response to cisplatin and paclitaxel chemotherapy as well as prognosis in NSCLC patients. However, the role of Pokemon in NSCLC treated with other chemotherapy regimens remain unclear.

Thirdly, we demonstrated that there was a poor response and shorter survival time in patients with a pretreatment Pokemon level $\geq 135.09 \mathrm{ng} / \mathrm{ml}$, which revealed Pokemon may exerted its characteristic resistance to chemotherapy. Thus, the molecular mechanism underlying Pokemon's involvement in resistance to chemotherapy remains to be further investigated.

Finally, the sample size in this study was very small, and further research with larger samples is warranted. In addition, more highquality, multicenter, large-sample, randomized controlled clinical trials are required to confirm our findings.

In conclusion, we showed that Pokemon expression levels were significantly higher in the serum of NSCLC patients compared to healthy controls. The pretreatment Pokemon level may identify patients with poor response to chemotherapy and poor prognosis, and was shown to be an independent risk factor for prognosis in patients with NSCLC. However, further studies are needed to verify these conclusions.

\section{Online Supplemental Material}

Fig. 1. Receiver operator characteristics (ROC) curve for pretreatment Pokemon levels in patients with non-small cell lung cancer (NSCLC). The area under the ROC curve was 0.831 (95\% confidence interval 0.675-0.987).

Fig. 2. The cumulative survival curves for non-small cell lung cancer (NSCLC) patients with Pokemon levels $<135.09 \mathrm{ng} / \mathrm{ml}$ and $\geq 135.09 \mathrm{ng} / \mathrm{ml}$. The survival of patients with Pokemon levels $<135.09 \mathrm{ng} / \mathrm{ml}$ was significantly longer than that of those with Pokemon levels $\geq 135.09 \mathrm{ng} / \mathrm{ml}$ (log-rank test, $\mathrm{p}=0.013$ )

To access the online supplemental material, please refer to www.karger. $\mathrm{com} /$ ?DOI=440790. 


\section{Acknowledgement}

We gratefully acknowledge Mr Wei-min Li, Zhi-yuan Gao, Zi-nan Feng, and Wen Gong for technical assistance. We thank Ms San-xiang Liu, Yue-ying $\mathrm{Qi}, \mathrm{Li} \mathrm{Li}$, and $\mathrm{Di} \mathrm{Xu}$ for advice and discussions.

\section{Disclosure Statement}

The authors declare no conflict of interest.

\section{References}

1 Maeda T, Hobbs RM, Merghoub T, et al.: Role of the proto-oncogene Pokemon in cellular transformation and ARF repression. Nature 2005;433:278-285.

$\checkmark 2$ Maeda T, Hobbs RM, Pandolfi PP: The transcription factor Pokemon: a new key player in cancer pathogenesis. Cancer Res 2005;65:8575-8577.

$\checkmark 3$ Kelly KF, Daniel JM: POZ for effect - POZ-ZF transcription factors in cancer and development. Trends Cell Biol 2006;16:578-587.

4 Stogios PP, Chen L, Prive GG: Crystal structure of the BTB domain from the LRF/ZBTB7 transcriptional regulator. Protein Sci 2007;16:336-342.

5 Guo C, Zhu K, Sun W, et al.: The effect of Pokemon on bladder cancer epithelial-mesenchymal transition. Biochem Biophys Res Commun 2014;443:1226-1231.
Zhao GT, Yang LJ, Li XX, et al.: Expression of the proto-oncogene Pokemon in colorectal cancer - inhibitory effects of an siRNA. Asian Pac J Cancer Prev 2013;14:4999-5005.

7 Aggarwal H, Aggarwal A, Agrawal DK: Epidermal growth factor increases LRF/Pokemon expression in human prostate cancer cells. Exp Mol Pathol 2011;91: 496-501.

$>8 \mathrm{Zu} \mathrm{X}, \mathrm{Ma}$ J, Liu H, et al.: Pro-oncogene Pokemon promotes breast cancer progression by upregulating survivin expression. Breast Cancer Res 2011;13:R26.

$\checkmark 9$ Jin XL, Sun QS, Liu F, et al.: microRNA 21-mediated suppression of Sproutyl by Pokemon affects liver cancer cell growth and proliferation. J Cell Biochem 2013; 114:1625-1633.
10 Apostolopoulou K, Pateras IS, Evangelou K, et al. Gene amplification is a relatively frequent event leading to ZBTB7A (Pokemon) overexpression in nonsmall cell lung cancer. J Pathol 2007;213:294-302.

11 Zhao ZH, Wang SF, Yu L, et al.: Expression of transcription factor Pokemon in non-small cell lung cancer and its clinical significance. Chin Med J (Engl) 2008; 121:445-449.

12 Bonomi P, Kim K, Fairclough D, et al.: Comparison of survival and quality of life in advanced non-small-cell lung cancer patients treated with two dose levels of paclitaxel combined with cisplatin versus etoposide with cisplatin: results of an Eastern Cooperative Oncology Group trial. J Clin Oncol 2000;18:623-631. 